\title{
Tauberian Theorems for Statistical Logarithmic Summability of Strongly Measurable Fuzzy Valued Functions
}

\author{
Özer Talo ${ }^{1}$, Enes Yavuz ${ }^{2 *}$, Hüsamettin Çoşkun ${ }^{3}$
}

\begin{abstract}
We define statistical logarithmic summability of strongly measurable fuzzy valued functions and we give slowly decreasing type Tauberian conditions under which statistical limit at infinity and statistical logarithmic summability of strongly measurable fuzzy valued functions imply ordinary limit at infinity in one dimensional fuzzy number space $E^{1}$. Besides, we give slowly oscillating type Tauberian conditions for statistical limit and statistical logarithmic summability of strongly measurable fuzzy valued functions in $n$-dimensional fuzzy number space $E^{n}$.

Keywords: fuzzy set theory, logarithmic summability, statistical limits, Tauberian theorems 2010 AMS: 03E72,40A35,40G15,40E05
\end{abstract}

${ }^{1}$ Manisa Celal Bayar Üniversitesi Küme evleri, Yunusemre, Manisa, Turkey, ORCID: 0000-0003-1393-5414

${ }^{2}$ Department of Mathematics, Manisa Celal Bayar University, Manisa, Turkey, ORCID: 0000-0002-4335-5210

${ }^{3}$ Department of Mathematics, Manisa Celal Bayar University, Manisa, Turkey, ORCID: 0000-0002-2344-9682

*Corresponding author: enes.yavuz@cbu.edu.tr

Received: 26 April 2020, Accepted: 15 June 2020, Available online: 30 June 2020

\section{Preliminaries}

Let $\mathscr{K}_{c}\left(\mathbb{R}^{n}\right)$ denote the family of all nonempty compact convex subsets of $\mathbb{R}^{n}$. If $A, B \in \mathscr{K}_{c}\left(\mathbb{R}^{n}\right)$ and $k \in \mathbb{R}$ then the operations of addition and scalar multiplication are defined as

$$
A+B=\{a+b: a \in A, b \in B\} \text { and } k A=\{k a: a \in A\} .
$$

The Hausdorff metric on $\mathscr{K}_{c}\left(\mathbb{R}^{n}\right)$ is defined by

$$
d(A, B)=\max \left\{\sup _{a \in A} \inf _{b \in B}\|a-b\|, \sup _{b \in B} \inf _{a \in A}\|a-b\|\right\},
$$

where $\|$.$\| denotes the usual Euclidean norm in \mathbb{R}^{n}$.

A fuzzy number is a mapping $u: \mathbb{R}^{n} \rightarrow[0,1]$ which satisfies the following four conditions:

(i) $u$ is normal, i.e. there exists an $x_{0} \in \mathbb{R}^{n}$ such that $u\left(x_{0}\right)=1$.

(ii) $u$ is fuzzy convex, i.e. $u[\lambda x+(1-\lambda) y] \geq \min \{u(x), u(y)\}$, for all $x, y \in \mathbb{R}^{n}$ and for all $\lambda \in[0,1]$.

(iii) $u$ is upper semi-continuous.

(iv) The set $[u]_{0}:=\overline{\left\{x \in \mathbb{R}^{n}: u(x)>0\right\}}$ is compact.[1]

This work was presented partly in 4th International Conference on Pure and Applied Sciences(Renewable Energy), ICPAS2017, November 23-25, 2017, Istanbul-Turkey with title "On some statistical weighted mean summability methods of fuzzy-number-valued functions". 
The set of all fuzzy numbers is denoted by $E^{n}$ and $E^{n}$ is called fuzzy number space. If $u \in E^{n}$, then $\alpha$-level set $[u]_{\alpha}$ of $u$, defined by

$$
[u]_{\alpha}:=\left\{\begin{array}{lc}
\left\{x \in \mathbb{R}^{n}: u(x) \geq \alpha\right\} & , \quad(0<\alpha \leq 1), \\
\left\{x \in \mathbb{R}^{n}: u(x)>0\right\} & , \quad(\alpha=0),
\end{array}\right.
$$

is a nonempty compact convex subset of $\mathbb{R}^{n}$.

Let $r \in \mathbb{R}^{n}$. We say that $\bar{r}$ is a crisp fuzzy number if

$$
\bar{r}(x):=\left\{\begin{array}{ccc}
1 & , & \text { if } x=r \\
0 & , & \text { otherwise. }
\end{array}\right.
$$

The operations addition and scalar multiplication on fuzzy numbers are defined by

$$
u+v=w \quad \Longleftrightarrow \quad[w]_{\alpha}=[u]_{\alpha}+[v]_{\alpha}, \text { for all } \alpha \in[0,1]
$$

and

$$
[k u]_{\alpha}=k[u]_{\alpha}, \text { for all } \alpha \in[0,1] .
$$

Lemma 1.1. [2]

(i) $\overline{0} \in E^{n}$ is neutral element with respect to + , i.e., $u+\overline{0}=\overline{0}+u=u$, for all $u \in E^{n}$.

(ii) For any $a, b \in \mathbb{R}$ with $a, b \geq 0$ or $a, b \leq 0$, and any $u \in E^{n}$, we have $(a+b) u=a u+$ bu. For general $a, b \in \mathbb{R}$, the above property does not hold.

(iii) For any $a \in \mathbb{R}$ and any $u, v \in E^{n}$, we have $a(u+v)=a u+a v$.

(iv) For any $a, b \in \mathbb{R}$ and any $u \in E^{n}$, we have $a(b u)=(a b) u$.

The metric $D$ on $E^{n}$ is defined as follows:

$$
D(u, v):=\sup _{\alpha \in[0,1]} d\left([u]_{\alpha},[v]_{\alpha}\right) .
$$

From [2], we have the following lemma.

Lemma 1.2. Let $u, v, w, z \in E^{n}$ and $k \in \mathbb{R}$.

(i) $\left(E^{n}, D\right)$ is a complete metric space.

(ii) $D(k u, k v)=|k| D(u, v)$.

(iii) $D(u+v, w+v)=D(u, w)$.

(iv) $D(u+v, w+z) \leq D(u, w)+D(v, z)$.

(v) $|D(u, \overline{0})-D(v, \overline{0})| \leq D(u, v) \leq D(u, \overline{0})+D(v, \overline{0})$.

We recall the concepts of measurability and integrability for fuzzy valued function.

Definition 1.3. [3] Let $T=[a, b] \subset \mathbb{R}$. A function $s: T \rightarrow E^{n}$ is strongly measurable iffor all $\alpha \in[0,1]$ the set valued function $s_{\alpha}: T \rightarrow \mathscr{K}_{c}\left(\mathbb{R}^{n}\right)$ defined by

$$
s_{\alpha}(x)=[s(x)]_{\alpha}
$$

is Lebesgue measurable, when $\mathscr{K}_{c}\left(\mathbb{R}^{n}\right)$ is endowed with the topology generated by Hausdorff metric $d$.

Theorem 1.4. [3] If fuzzy valued function s is strongly measurable, then it is measurable with respect to the topology generated by $D$.

Definition 1.5. [3] Let $s: T \rightarrow E^{n}$. The integral of s over $T$ is defined by the following:

$$
\left[\int_{T} s(x) d x\right]_{\alpha}=\int_{T}[s(x)]_{\alpha} d x=\left\{\int_{T} f(x) d x \mid f: T \rightarrow \mathbb{R}^{n} \text { is a measurable selection of } s_{\alpha}\right\},
$$

for $\alpha \in(0,1]$. 
A function $s: T \rightarrow E^{n}$ is called integrably bounded if there exists an integrable function $h: T \rightarrow \mathbb{R}^{+}$such that $D(s(t), \overline{0}) \leq$ $h(t)$, for all $t \in T$.

A strongly measurable and integrably bounded function $s: T \rightarrow E^{n}$ is said to be integrable over $T$ if

$$
\int_{T} s(x) d x \in E^{n}
$$

Theorem 1.6. [3] If $s: T \rightarrow E^{n}$ is strongly measurable and integrably bounded, then s is integrable.

Definition 1.7. A fuzzy valued function $s: T \rightarrow E^{n}$ is said to be continuous at $x_{0} \in T$ if for each $\varepsilon>0$ there is a $\delta>0$ such that $D\left(s(x), s\left(x_{0}\right)\right)<\varepsilon$, whenever $x \in T$ with $\left|x-x_{0}\right|<\delta$. If $s$ is continuous at each $x \in T$, then we say $s$ is continuous on $T$.

Theorem 1.8. [3] If $f: T \rightarrow E^{n}$ is continuous then it is strongly measurable.

Strong measurability of fuzzy valued functions does not imply continuity by the following example.

Example 1.9. Let $\mu, v \in E^{n}$ with $\mu \neq v$ and define $s: T \rightarrow E^{n}$ by

$$
s(x):= \begin{cases}\mu, & \text { if } x \in \mathbb{Q} \\ v, & \text { otherwise. }\end{cases}
$$

Fuzzy valued function s is strongly measurable but it is not continuous.

Theorem 1.10. [3] If $s: T \rightarrow E^{n}$ is continuous, then $s$ is integrable.

Theorem 1.11. [3] If $s: T \rightarrow E^{n}$ is continuous, $g(x)=\int_{a}^{x} s(t) d t$ is Lipschitz continuous on $T$.

Theorem 1.12. [3] Let $f, g: T \rightarrow E^{n}$ be integrable and $\lambda \in \mathbb{R}$. Then,

(i) $\int_{T}(f(x)+g(x)) d x=\int_{T} f(x) d x+\int_{T} g(x) d x$;

(ii) $\int_{T} \lambda f(x) d x=\lambda \int_{T} f(x) d x$;

(iii) $\int_{a}^{b} f(x) d x=\int_{a}^{c} f(x) d x+\int_{c}^{b} f(x) d x$, where $a<c<b$.

(iv) The function $F: T \rightarrow \mathbb{R}_{+}$defined by $F(x)=D(f(x), g(x))$ is integrable on $T$ and

$$
D\left(\int_{T} f(x) d x, \int_{T} g(x) d x\right) \leq \int_{T} D(f(x), g(x)) d x .
$$

Lemma 1.13. [2] Suppose $\mu \in E^{n}$ and define $s: T \rightarrow E^{n}$ by $s(x)=\mu$, for all $x \in[a, b]$. Then,

$$
\int_{a}^{b} s(x) d x=(b-a) \mu \text {. }
$$

If $u \in E^{1}$, then $\alpha$-level set $[u]_{\alpha}$ of $u$ is closed, bounded and non-empty interval and we can write $[u]_{\alpha}:=\left[u^{-}(\alpha), u^{+}(\alpha)\right]$. The partial ordering relation on $E^{1}$ is defined as follows:

$$
u \preceq v \Longleftrightarrow[u]_{\alpha} \preceq[v]_{\alpha} \Longleftrightarrow u^{-}(\alpha) \leq v^{-}(\alpha) \text { and } u^{+}(\alpha) \leq v^{+}(\alpha) \text {, for all } \alpha \in[0,1] .
$$

Combining the results of Lemma 6 in [4], Lemma 5 in [5], Lemma 3.4, Theorem 4.9 in [6] and Lemma 14 in[7], following lemma is obtained.

Lemma 1.14. Let $u, v, w, e \in E^{1}$ and $\varepsilon>0$. The following statements hold:

(i) $D(u, v) \leq \varepsilon$ if and only if $u-\bar{\varepsilon} \preceq v \preceq u+\bar{\varepsilon}$

(ii) If $u \preceq v+\bar{\varepsilon}$ for every $\varepsilon>0$, then $u \preceq v$.

(iii) If $u \preceq v$ and $v \preceq w$, then $u \preceq w$.

(iv) If $u \preceq w$ and $v \preceq e$, then $u+v \preceq w+e$.

(v) If $u+w \preceq v+w$ then $u \preceq v$. 
A fuzzy valued function $s: T \rightarrow E^{1}$ has the parametric representation

$$
[s(x)]_{\alpha}=\left[s_{\alpha}^{-}(x), s_{\alpha}^{+}(x)\right]
$$

where $s_{\alpha}^{+}, s_{\alpha}^{-}: T \rightarrow \mathbb{R}$, for all $\alpha \in[0,1]$.

Theorem 1.15. [8] Fuzzy valued function $s: T \rightarrow E^{1}$ is strongly measurable if and only if $s_{\alpha}^{+}$and $s_{\alpha}^{-}$are measurable for all $\alpha \in[0,1]$.

Lemma 1.16. [9] Fuzzy valued function $s: T \rightarrow E^{1}$ is integrable if and only if $s_{\alpha}^{+}, s_{\alpha}^{-}$are integrable over $T$ and

$$
\left[\int_{T} s(x) d x\right]_{\alpha}=\left[\int_{T} s_{\alpha}^{-}(x) d x, \int_{T} s_{\alpha}^{+}(x) d x\right],
$$

for all $\alpha \in[0,1]$.

Lemma 1.17. [9] Let $f, g: T \rightarrow E^{1}$ be integrable and $f(x) \preceq g(x)$, for all $x \in T$. Then, $\int_{T} f(x) d x \preceq \int_{T} g(x) d x$.

Talo et al.[9] and Belen[10] defined statistical limits of fuzzy valued functions at $\infty$ independently. Talo et al.[9] took the case of strongly measurable fuzzy valued functions while Belen[10] took the case of continuous fuzzy valued functions. In view of Theorem 1.8 and Example 1.9, definition of Talo et al.[9] is more general and hence we prefer to use that definiton.

Definition 1.18. [9] A strongly measurable fuzzy valued function $s:[a, \infty) \rightarrow E^{n}$ has statistical limit at $\infty$ if there exists a fuzzy number $\mu$ such that for every $\varepsilon>0$,

$$
\lim _{b \rightarrow \infty} \frac{1}{b-a}|\{x \in(a, b): D(s(x), \mu)>\varepsilon\}|=0,
$$

where by $|\{\}$.$| we denote the Lebesgue measure of the set \{$.$\} . In this case, we write s t-\lim _{x \rightarrow \infty} s(x)=\mu$.

Remark 1.19. In (1.2), the set $\{x \in(a, b): D(s(x), \mu)>\varepsilon\}$ is Lebesgue measurable by Theorem 1.4.

Theorem 1.20. [9] Let s be strongly measurable fuzzy valued function. Then,

$$
\lim _{x \rightarrow \infty} s(x)=\mu \Rightarrow s t-\lim _{x \rightarrow \infty} s(x)=\mu \text {. }
$$

The converse of Theorem 1.20 does not hold in general. As a counter example, we can give Example 1.9.

Definition 1.21. [11] A fuzzy valued function $s:[1, \infty) \rightarrow E^{1}$ is said to be slowly decreasing with respect to logarithmic summability if for every $\varepsilon>0$ there exist $x_{0}>1$ and $\lambda>1$ such that

$$
s(t) \succeq s(x)-\bar{\varepsilon}
$$

whenever $x_{0} \leq x<t \leq x^{\lambda}$.

Definition 1.22. A fuzzy valued function $s:[1, \infty) \rightarrow E^{n}$ is said to be slowly oscillating with respect to logarithmic summability if for every $\varepsilon>0$ there exist $x_{0}>1$ and $\lambda>1$ such that

$$
D(s(t), s(x)) \leq \varepsilon
$$

whenever $x_{0} \leq x<t \leq x^{\lambda}$.

\section{Main Results}

By $L_{l o c}\left([a, \infty), E^{n}\right)$, we denote the set of fuzzy valued functions $s:[a, \infty) \rightarrow E^{n}$ such that $s$ is integrable on every bounded interval $[a, x], x>a$. We define statistical logarithmic summability of fuzzy valued functions as the following.

Definition 2.1. Let $s \in L_{l o c}\left([1, \infty), E^{n}\right)$. Logarithmic average $\tau(x)$ of $s$ is defined by

$$
\tau(x)=\frac{1}{\log x} \int_{1}^{x} \frac{s(u)}{u} d u, \quad x \in(1, \infty) .
$$

We say that $s$ is statistically logarithmic summable to a fuzzy number $\mu$ if $s_{x \rightarrow \infty}-\lim \tau(x)=\mu$. 
By [11, Theorem 3.2] and Theorem 1.20, ordinary limit of fuzzy valued functions at infinity implies statistical logarithmic summability. But the converse is not true in general which can be seen by the following example.

Example 2.2. Take fuzzy valued function $s:[1, \infty) \rightarrow E^{1}$ such that

$$
(s(x))(t)=\left\{\begin{array}{lll}
1-(t-\sin x)^{2}, & \text { if } & \sin x-1 \leq t \leq \sin x+1 \\
0, & \text { otherwise }
\end{array}\right.
$$

Then, we have

$$
\begin{aligned}
& s_{\alpha}^{-}(x)=\sin x-\sqrt{1-\alpha}, s_{\alpha}^{+}(x)=\sin x+\sqrt{1-\alpha} \\
& \lim _{x \rightarrow \infty} \tau_{\alpha}^{-}(x)=\lim _{x \rightarrow \infty} \frac{1}{\log x} \int_{1}^{x} \frac{s_{\alpha}^{-}(u)}{u} d u=-\sqrt{1-\alpha} \\
& \lim _{x \rightarrow \infty} \tau_{\alpha}^{+}(x)=\lim _{x \rightarrow \infty} \frac{1}{\log x} \int_{1}^{x} \frac{s_{\alpha}^{+}(u)}{u} d u=\sqrt{1-\alpha}
\end{aligned}
$$

Since $\lim _{x \rightarrow \infty} D(\tau(x), \mu)=0$ where $[\mu]_{\alpha}=[-\sqrt{1-\alpha}, \sqrt{1-\alpha}]$, by Theorem 1.20 fuzzy valued function s is statistical logarithmic summable to fuzzy number

$$
\mu(t)= \begin{cases}1-t^{2} & \text { if } \quad-1 \leq t \leq 1 \\ 0 & \text { otherwise }\end{cases}
$$

But ordinary limit of s at infinity does not exist.

In the following two theorems, we give slowly decreasing and slowly oscillating type conditions under which statistical limits of fuzzy valued functions implies ordinary limit at infinity.

Theorem 2.3. If a strongly measurable fuzzy valued function $s:[1, \infty) \rightarrow E^{1}$ is slowly decreasing with respect to logarithmic summability, then $s t$ - $\lim _{x \rightarrow \infty} s(x)=\mu$ implies $\lim _{x \rightarrow \infty} s(x)=\mu$.

Proof. Let strongly measurable fuzzy valued function $s:[1, \infty) \rightarrow E^{1}$ be slowly decreasing with respect to logarithmic summability and st-lim $s(x)=\mu$. Then for given an $\varepsilon>0$ there exist $x_{0}>1$ and $\lambda>1$ such that slow decrease condition (1.4) is satisfied. Also, as in [12, Proof of Theorem 1], since $\operatorname{st}_{x \rightarrow \infty} s(x)=\mu$ there exists a sequence $b_{n} \uparrow \infty$ of real numbers such that

$$
D\left(s\left(b_{n}\right), \mu\right) \leq \varepsilon, \quad n=1,2, \ldots
$$

and for some $n_{0}$ we have

$$
b_{n+1}<b_{n}^{\lambda}, \quad n=n_{0}+1, n_{0}+2, \ldots
$$

Now consider $t \in\left(b_{n}, b_{n+1}\right]$ for $n>n_{0}$. In view of (2.2) and monotonicity of sequence $\left(b_{n}\right)$ we get

$$
b_{n}<t \leq b_{n+1}<b_{n}^{\lambda}<t^{\lambda} .
$$

So by slow decrease condition (1.4) and by (2.1), for every $n>n_{0}$ and $t \in\left(b_{n}, b_{n+1}\right]$ we have

$$
s(t) \succeq s\left(b_{n}\right)-\bar{\varepsilon} \succeq \mu-2 \bar{\varepsilon} .
$$

Again for every $n>n_{0}$ and $t \in\left(b_{n}, b_{n+1}\right]$ we have

$$
s(t) \preceq s\left(b_{n+1}\right)+\bar{\varepsilon} \preceq \mu+2 \bar{\varepsilon} .
$$

Then combining (2.3) and (2.4) we get

$$
D(s(t), \mu) \leq 2 \varepsilon \quad \text { for every } \quad t \in \bigcup_{n=n_{0}+1}^{\infty}\left(b_{n}, b_{n+1}\right]=\left(b_{n_{0}+1}, \infty\right) .
$$

This proves $\lim _{x \rightarrow \infty} s(x)=\mu$.

Theorem 2.4. If a strongly measurable fuzzy valued function $s:[1, \infty) \rightarrow E^{n}$ is slowly oscillating with respect to logarithmic summability, then $\operatorname{st}_{x \rightarrow \infty} \lim _{x \rightarrow \infty} s(x)=\mu$ implies $\lim _{x \rightarrow \infty} s(x)=\mu$. 
Proof. Let st-lim $s(x)=\mu$ and $s$ be slowly oscillating with respect to logarithmic summability. Then as in the proof of Theorem 2.3 , for given $\varepsilon>0$ and $\lambda>1$ there exists a sequence $b_{n} \uparrow \infty$ such that (2.1) and (2.2) are satisfied. By condition (2.2) and by condition of slow oscillation we have

$$
D\left(s(t), s\left(b_{n}\right)\right) \leq \varepsilon \quad \text { whenever } \quad x_{0} \leq b_{n}<t<b_{n+1}
$$

for large enough $n$, say $n>n_{1}$. From (2.1) and (2.5) it follows that

$$
D(s(t), \mu) \leq D\left(s(t), s\left(b_{n}\right)\right)+D\left(s\left(b_{n}\right), \mu\right) \leq 2 \varepsilon
$$

for every $t \in \bigcup_{n=n_{1}+1}^{\infty}\left(b_{n}, b_{n+1}\right]=\left(b_{n_{1}+1}, \infty\right)$. This means that $\lim _{x \rightarrow \infty} s(x)=\mu$.

Now we aim to replace logarithmic summability with statistical logarithmic summability in Theorem 2.8.

Lemma 2.5. If $s:[1, \infty) \rightarrow E^{1}$ is a fuzzy valued function such that slow decrease condition with respect to logarithmic summability (1.4) is satisfied for $\varepsilon:=1$ where $x_{0}>1$ and $\lambda>1$, then there exists a constant $B_{1}>0$ such that

$$
s(t) \succeq s(x)-B_{1} \ln \left(\frac{\ln t}{\ln x}\right) \quad \text { whenever } \quad x_{0} \leq x<t^{1 / \lambda}
$$

Proof. Let $s:[1, \infty) \rightarrow E^{1}$ be a fuzzy valued function such that slow decrease condition with respect to logarithmic summability (1.4) is satisfied only for $\varepsilon:=1$ where $x_{0}>1$ and $\lambda>1$, and let $x_{0} \leq x<t^{1 / \lambda}$ be given. Then consider the sequence

$$
t_{0}:=t, \quad t_{p}:=t_{p-1}^{1 / \lambda}, \quad p=1,2, \ldots, q+1,
$$

where $q$ is defined by the condition $t_{q+1} \leq x<t_{q}$. Since (1.4) is satisfied for $\varepsilon:=1$, we get

$$
s(t) \succeq s\left(t_{1}\right)-1 \succeq s\left(t_{2}\right)-2 \succeq \cdots \succeq s\left(t_{q}\right)-q \succeq s(x)-q-1 .
$$

Then by the calculations regarding $q$ in [12, Proof of Lemma 1], we get

$$
s(t) \succeq s(x)-1-\frac{1}{\ln \lambda} \ln \left(\frac{\ln t}{\ln x}\right) \quad \text { whenever } \quad x_{0} \leq x<t^{1 / \lambda} .
$$

Then in view of $x<t^{1 / \lambda}$, we have $\ln \lambda<\ln \left(\frac{\ln t}{\ln x}\right)$ and as result we conclude

$$
s(t) \succeq s(x)-B_{1} \ln \left(\frac{\ln t}{\ln x}\right) \quad \text { whenever } \quad x_{0} \leq x<t^{1 / \lambda}
$$

with $B_{1}:=2 / \ln \lambda$.

Lemma 2.6. $s \in L_{l o c}\left([1, \infty), E^{1}\right)$. Under the assumptions of Lemma 2.5 , there exists a constant $B_{2}>0$ such that

$$
\frac{1}{\ln t} \int_{x_{0}}^{t} \frac{s(t)}{x} d x \succeq \frac{1}{\ln t} \int_{x_{0}}^{t} \frac{s(x)}{x} d x-B_{2} \quad \text { whenever } \quad t>x_{0}^{\lambda} .
$$

Proof. Let the fuzzy valued function $s$ satisfy slow decrease condition only for $\varepsilon:=1$ where this time assume $x_{0}>e$. Then by (2.6), we get the following:

$$
\begin{aligned}
\int_{x_{0}}^{t} \frac{s(t)}{x} d x & =\int_{x_{0}}^{t^{1 / \lambda}} \frac{s(t)}{x} d x+\int_{t^{1 / \lambda}}^{t} \frac{s(t)}{x} d x \\
& \succeq \int_{x_{0}}^{t^{1 / \lambda}} \frac{s(x)}{x} d x-B_{3} \int_{x_{0}}^{t^{1 / \lambda}} \frac{1}{x} \ln \left(\frac{\ln t}{\ln x}\right) d x+\int_{t^{1 / \lambda}}^{t} \frac{s(x)}{x} d x-\int_{t^{1 / \lambda}}^{t} \frac{d x}{x} \\
& =\int_{x_{0}}^{t} \frac{s(x)}{x} d x-B_{3} \int_{x_{0}}^{t^{1 / \lambda}} \frac{1}{x} \ln \left(\frac{\ln t}{\ln x}\right) d x-\int_{t^{1 / \lambda}}^{t} \frac{d x}{x} \\
& \succeq \int_{x_{0}}^{t} \frac{s(x)}{x} d x-B_{3}(\ln t)\left(\frac{\ln \lambda}{\lambda}+\frac{\left(\ln \ln x_{0}\right)}{\lambda}+\frac{1}{\lambda}\right) .
\end{aligned}
$$

where we took into account the calculations in [12, Proof of Lemma 3]. If we take

$$
B_{2}:=\frac{B_{1}}{\lambda}\left(\ln \lambda+\ln \ln x_{0}+1\right)
$$

this proves (2.8). 
Lemma 2.7. If $s \in L_{l o c}\left([1, \infty), E^{1}\right)$ is slowly decreasing with respect to logarithmic summability, then logarithmic mean $\tau$ is slowly decreasing with respect to logarithmic summability.

Proof. Let $s \in L_{l o c}\left([1, \infty), E^{1}\right)$ and be $s$ be slowly decreasing with respect to logarithmic summability. We aim to show that logarithmic mean $\tau$ of $s$ is also slowly decreasing.

Let some $0<\varepsilon<1$ be given. Then consider $x_{0} \leq x<t \leq x^{\lambda}$ that in slow decrease condition (1.4), where

$$
1<\lambda \leq 1+\frac{\varepsilon}{\max \left\{1, B_{2}\right\}}
$$

and $B_{2}$ is from (2.8).

By the following equality

$$
\begin{aligned}
& \tau(t)+\left(1-\frac{\ln x}{\ln t}\right) \frac{1}{\ln x} \int_{1}^{x_{0}} \frac{s(u)}{u} d u+\left(1-\frac{\ln x}{\ln t}\right) \frac{1}{\ln x} \int_{x_{0}}^{x} \frac{s(u)}{u} d u+\left(1-\frac{\ln x}{\ln t}\right) \frac{\ln x_{0}}{\ln x} s(x) \\
& =\tau(t)+\left(1-\frac{\ln x}{\ln t}\right) \tau(x)+\left(1-\frac{\ln x}{\ln t}\right) \frac{\ln x_{0}}{\ln x} s(x) \\
& =\tau(x)+\frac{1}{\ln t} \int_{x}^{t} \frac{s(u)}{u} d u+\left(1-\frac{\ln x}{\ln t}\right) \frac{\ln x_{0}}{\ln x} s(x),
\end{aligned}
$$

we have

$$
\begin{aligned}
& \tau(t)+\left(1-\frac{\ln x}{\ln t}\right) \frac{1}{\ln x} \int_{1}^{x_{0}} \frac{s(u)}{u} d u+\left(1-\frac{\ln x}{\ln t}\right) \frac{1}{\ln x} \int_{x_{0}}^{x} \frac{s(u)}{u} d u+\left(1-\frac{\ln x}{\ln t}\right) \frac{\ln x_{0}}{\ln x} s(x) \\
& =\tau(x)+\frac{1}{\ln t} \int_{x}^{t} \frac{s(u)}{u} d u+\left(1-\frac{\ln x}{\ln t}\right) \frac{\ln x_{0}}{\ln x} s(x) .
\end{aligned}
$$

Then by Lemma 2.6 and from slow decrease condition (1.4) we get

$$
\begin{aligned}
& \tau(t)+\left(1-\frac{\ln x}{\ln t}\right) \frac{1}{\ln x} \int_{1}^{x_{0}} \frac{s(u)}{u} d u+\left(1-\frac{\ln x}{\ln t}\right)\left\{\frac{1}{\ln x} \int_{x_{0}}^{x} \frac{s(x)}{u} d u+B_{2}\right\}+\left(1-\frac{\ln x}{\ln t}\right) \frac{\ln x_{0}}{\ln x} s(x) \\
& \succeq \tau(x)+\frac{1}{\ln t} \int_{x}^{t} \frac{s(x)-1}{u} d u+\left(1-\frac{\ln x}{\ln t}\right) \frac{\ln x_{0}}{\ln x} s(x),
\end{aligned}
$$

which yields

$$
\tau(t)+\left(1-\frac{\ln x}{\ln t}\right) \frac{1}{\ln x} \int_{1}^{x_{0}} \frac{s(u)}{u} d u+\left(1-\frac{\ln x}{\ln t}\right) B_{2} \succeq \tau(x)-\left(1-\frac{\ln x}{\ln t}\right)+\left(1-\frac{\ln x}{\ln t}\right) \frac{\ln x_{0}}{\ln x} s(x) .
$$

At this point there exists $x_{1}>x_{0}^{\lambda}$ such that

$$
\left(1-\frac{\ln x}{\ln t}\right) \frac{\ln x_{0}}{\ln x} s(x) \succeq-\bar{\varepsilon} \quad \text { whenever } \quad x>x_{1}
$$

holds since by (2.7) we have

$$
\frac{s(x)}{\ln x} \succeq \frac{s\left(x_{0}\right)-1}{\ln x}-\frac{1}{\ln x \ln \lambda} \ln \left(\frac{\ln x}{\ln x_{0}}\right) \rightarrow 0 \text { as } x \rightarrow \infty .
$$

Besides there exists $x_{2}$ such that

$$
\left(1-\frac{\ln x}{\ln t}\right) \frac{1}{\ln x} \int_{1}^{x_{0}} \frac{s(u)}{u} d u \preceq-\bar{\varepsilon} \quad \text { whenever } \quad x>x_{2},
$$

since

$$
\lim _{x \rightarrow \infty}\left(1-\frac{\ln x}{\ln t}\right) \frac{1}{\ln x} \int_{1}^{x_{0}} \frac{s(u)}{u} d u=\overline{0} .
$$

Also from the fact $\frac{1}{\lambda} \leq \frac{\ln x}{\ln t}$ we have

$$
\left(1-\frac{\ln x}{\ln t}\right) B_{2} \leq\left(1-\frac{1}{\lambda}\right) B_{2} \leq(\lambda-1) B_{2} \leq \varepsilon,
$$


and again from the fact that $\frac{1}{\lambda} \leq \frac{\ln x}{\ln t}$ we get

$$
-\left(1-\frac{\ln x}{\ln t}\right) \geq-\left(1-\frac{1}{\lambda}\right) \geq-(\lambda-1) \geq-\varepsilon .
$$

Then inserting the expressions (2.11)-(2.14) in equality (2.10) we get

$$
\tau(t) \succeq \tau(x)-4 \bar{\varepsilon} \quad \text { whenever } \quad x_{3} \leq x<t \leq x^{\lambda},
$$

where $x_{3}=\max \left\{x_{0}, x_{1}, x_{2}\right\}$. This proves that $\tau$ is slowly decreasing with respect to logarithmic summability.

Analogous of Corollary 3.8 in [11] can be given as the following.

Theorem 2.8. If $s \in L_{l o c}\left([1, \infty), E^{n}\right)$ is logarithmic summable to a fuzzy number $\mu$ and is slowly decreasing with respect to logarithmic summability then $\lim _{x \rightarrow \infty} s(x)=\mu$.

In view of Theorem 2.8, Theorem 2.3 and Lemma 2.7 we give the following result.

Theorem 2.9. If $s \in L_{l o c}\left([1, \infty), E^{1}\right)$ is slowly decreasing with respect to logarithmic summability, then st- $\lim _{x \rightarrow \infty} \tau(x)=\mu$ implies $\lim _{x \rightarrow \infty} s(x)=\mu$.

Replacing absolute value with metric $D$ in Lemma 2 and Lemma 4 in [12] we obtain the following lemmas in fuzzy setting.

Lemma 2.10. If $s:[1, \infty) \rightarrow E^{n}$ is a fuzzy valued function such that slow oscillation condition with respect to logarithmic summability (1.5) is satisfied for $\varepsilon:=1$ where $x_{0}>1$ and $\lambda>1$, then there exists a constant $B_{3}>0$ such that

$$
D(s(t), s(x)) \leq B_{3} \ln \left(\frac{\ln t}{\ln x}\right) \quad \text { whenever } \quad x_{0} \leq x<t^{1 / \lambda}
$$

Lemma 2.11. Let $s \in L_{l o c}\left([1, \infty), E^{n}\right)$. Under the assumptions of Lemma 2.10, there exists a constant $B_{4}>0$ such that

$$
\frac{1}{\ln t} \int_{x_{0}}^{t} \frac{D(s(t), s(x))}{x} d x \leq B_{4} \quad \text { whenever } \quad t>x_{0}^{\lambda} .
$$

Lemma 2.12. If $s \in L_{l o c}\left([1, \infty), E^{n}\right)$ is slowly oscillating with respect to logarithmic summability, then logarithmic mean $\tau$ is also slowly oscillating.

Proof. As in the proof of Lemma 2.7, for given $0<\varepsilon<1$ consider $x_{0} \leq x<t \leq x^{\lambda}$ that in slow oscillation condition (1.5), where

$$
1<\lambda \leq 1+\frac{\varepsilon}{\max \left\{1, B_{4}\right\}}
$$

and $B_{4}$ is from Lemma 2.11. Adding $2\left(1-\frac{\ln x}{\ln t}\right)\left(1-\frac{\ln x_{0}}{\ln x}\right) s(x)$ to both sides of the equation (2.9) we get

$$
\begin{aligned}
& \tau(t)+\frac{\ln t-\ln x}{\ln t \ln x} \int_{1}^{x_{0}} \frac{s(u)}{u} d u+\frac{\ln t-\ln x}{\ln t \ln x} \int_{x_{0}}^{x} \frac{s(u)}{u} d u+\frac{1}{\ln t} \int_{x}^{t} \frac{s(x)}{u} d u+\frac{\ln t-\ln x}{\ln t \ln x}\left(\ln x-\ln x_{0}\right) s(x) \\
& =\tau(x)+\frac{1}{\ln t} \int_{x}^{t} \frac{s(u)}{u} d u+\frac{\ln t-\ln x}{\ln t} s(x)+\frac{\ln t-\ln x}{\ln t \ln x} \int_{x_{0}}^{x} \frac{s(x)}{u} d u .
\end{aligned}
$$

Then by the properties given in Lemma 1.2 and Theorem 1.12 we have

$$
\begin{aligned}
& D(\tau(t), \tau(x))= D\left(\frac{\ln t-\ln x}{\ln t \ln x} \int_{1}^{x_{0}} \frac{s(u)}{u} d u+\frac{\ln t-\ln x}{\ln t \ln x} \int_{x_{0}}^{x} \frac{s(u)}{u} d u+\frac{1}{\ln t} \int_{x}^{t} \frac{s(x)}{u} d u+\frac{\ln t-\ln x}{\ln t \ln x}\left(\ln x-\ln x_{0}\right) s(x),\right. \\
&\left.\frac{1}{\ln t} \int_{x}^{t} \frac{s(u)}{u} d u+\frac{\ln t-\ln x}{\ln t} s(x)+\frac{\ln t-\ln x}{\ln t \ln x} \int_{x_{0}}^{x} \frac{s(x)}{u} d u\right) \\
& \leq \quad \frac{\ln t-\ln x}{\ln t \ln x} \ln x_{0} D(s(x), \overline{0})+\frac{\ln t-\ln x}{\ln t \ln x} \int_{1}^{x_{0}} \frac{D(s(u), \overline{0})}{u} d u+\frac{\ln t-\ln x}{\ln t \ln x} \int_{x_{0}}^{x} \frac{D(s(u), s(x))}{u} d u \\
& \quad+\frac{1}{\ln t} \int_{x}^{t} \frac{D(s(u), s(x))}{u} d u \\
&=J_{1}+J_{2}+J_{3}+J_{4} .
\end{aligned}
$$


By Lemma 2.10, there exists $x_{1}>x_{0}^{\lambda}$ such that $J_{1} \leq \varepsilon$ for $x>x_{1}$ in view of the fact that

$$
\frac{D(s(x), \overline{0})}{\ln x} \leq \frac{D\left(s(x), s\left(x_{0}\right)\right)}{\ln x}+\frac{D\left(s\left(x_{0}\right), \overline{0}\right)}{\ln x} \leq B_{3} \frac{\ln \left(\ln x / \ln x_{0}\right)}{\ln x}+\frac{D\left(s\left(x_{0}\right), \overline{0}\right)}{\ln x} \rightarrow 0 \quad(\text { as } x \rightarrow \infty) .
$$

Besides, since

$$
\lim _{x \rightarrow \infty} \frac{\ln t-\ln x}{\ln t \ln x} \int_{1}^{x_{0}} \frac{D(s(u), \overline{0})}{u} d u=0
$$

there exists $x_{2}$ such that $J_{2} \leq \varepsilon$ for $x>x_{2}$.

Furthermore, from the fact that $\frac{1}{\lambda} \leq \frac{\ln x}{\ln t}$ and by Lemma 2.11 we have $J_{3} \leq(\lambda-1) B_{4} \leq \varepsilon$ for $x>x_{0}^{\lambda}$.

Again from the fact that $\frac{1}{\lambda} \leq \frac{\ln x}{\ln t}$ and by slow oscillation condition we have $J_{4} \leq \varepsilon$.

Hence combining all findings we have

$$
D(\tau(t), \tau(x)) \leq J_{1}+J_{2}+J_{3}+J_{4} \leq 4 \varepsilon \quad \text { whenever } \quad x_{3} \leq x<t \leq x^{\lambda},
$$

where $x_{3}=\max \left\{x_{1}, x_{2}\right\}$, and this completes the proof.

Analogous of Corollary 2.1 in [13] may be given for $s \in L_{l o c}\left([1, \infty), E^{n}\right)$ as the following. The proof is similar and hence omitted.

Theorem 2.13. If $s \in L_{l o c}\left([1, \infty), E^{n}\right)$ is logarithmic summable to a fuzzy number $\mu$ and is slowly oscillating with respect to logarithmic summability, then $\lim _{x \rightarrow \infty} s(x)=\mu$.

In view of Theorem 2.4, Lemma 2.12 and Theorem 2.13 we give the following result.

Theorem 2.14. If $s \in L_{l o c}\left([1, \infty), E^{n}\right)$ is slowly oscillating with respect to logarithmic summability, then $s t-\lim _{x \rightarrow \infty} \tau(x)=\mu$ implies $\lim _{x \rightarrow \infty} s(x)=\mu$.

\section{Acknowledgement}

We would like to thank reviewers for their careful reading and constructive criticism of the earlier version of this paper which improved the presentation and its readability.

\section{References}

[1] L. A. Zadeh, Fuzzy sets, Inform. Control 8(1965) 338-353.

[2] J. Li, A. Zhao and J. Yan, The Cauchy problem of fuzzy differential equations under generalized differentiability, Fuzzy Set Syst 200(2012) 1-24.

[3] O. Kaleva, Fuzzy differential equations, Fuzzy Set Syst 24(1987) 301-317.

[4] S. Aytar, M. Mammadov and S. Pehlivan, Statistical limit inferior and limit superior for sequences of fuzzy numbers, Fuzzy Set Syst 157(7) (2006) 976-985.

[5] S. Aytar and S. Pehlivan, Statistical cluster and extreme limit points of sequences of fuzzy numbers, Inform. Sci. 177(16) (2007) 3290-3296

[6] H. Li and C. Wu, The integral of a fuzzy mapping over a directed line, Fuzzy Set Syst 158(2007) 2317-2338.

[7] Ö. Talo and F. Başar, On the slowly decreasing sequences of fuzzy numbers, Abstr. Appl. Anal. 2013(2013) 1-7.

[8] Y. K. Kim and B. M. Ghil, Integrals of fuzzy-number-valued functions, Fuzzy Set Syst 86(1997) 213-222

[9] Ö. Talo, E. Yavuz and H. Çoşkun, On the statistical limits of strongly measurable fuzzy valued functions, Konuralp Journal of Mathematics 8(1) (2020), 62-69

[10] C. Belen, Tauberian theorems for statistical limit and statistical summability by weighted means of continuous fuzzy valued functions, J. Math. Ext. 14(4) (2020)

[11] E. Yavuz, H. Çoşkun, On the limits of logarithmic summable fuzzy-number-valued functions at infinity, Journal of Mathematical Analysis 8(3) (2017), 116-124.

[12] F. Móricz and Németh Z., Statistical extension of classical Tauberian theorems in the case of logarithmic summability, Anal. Math. 40(3) (2014) 231-242. 
Tauberian Theorems for Statistical Logarithmic Summability of Strongly Measurable Fuzzy Valued Functions -

[13] C. Belen, Tauberian theorems for weighted mean summability method of improper Riemann integrals of fuzzy-numbervalued functions, Soft Comput 22(12) (2018) 3951-3957 